\title{
Progress in Reactor and Accelerator Based BNCT at Kyoto University Research Reactor Institute
}

\author{
Yoshinori Sakurai ${ }^{1}$ \\ Kyoto University Research Reactor Institute \\ Asashiro-nishi 2-1010, Kumatori-cho, Sennan-gun, Osaka 590-0494, Japan \\ E-mail: yosakura@rri.kyoto-u.ac.jp
}

The world's first clinical irradiation for boron neutron capture therapy (BNCT) was carried out using a neutron irradiation field for BNCT, installed at a research nuclear reactor in USA in 1951. After this year until 2012, BNCT has been performed only using reactor-based irradiation systems. In Kyoto University Research Reactor Institute (KURRI), BNCT clinical study using Heavy Water Facility installed in Kyoto University Reactor (KUR) came to be regularly performed from February 1990. At first, BNCT in this institute was performed just for malignant brain tumor and melanoma. The application was extended for head and neck tumors in 2001, and for body tumors such as liver tumor, lung tumor, malignant pleural mesothelioma, etc. in 2005. There were the several interruption periods, but 510 clinical irradiations were carried out using KUR Heavy Water Facility as of June 2016. Concurrently with the clinical study using KUR Heavy Water Facility, the development of accelerator-based system has been studied. In early 2009, the world's first accelerator-based system for BNCT clinical irradiation, Cyclotron-Based Epi-thermal Neutron Source (C-BENS) was completed. The clinical trial using C-BENS was started in 2012. BNCT using various accelerator-based irradiation systems including C-BENS may be carried out at plural facilities in the near future. Thus, it is the time when BNCT is shifting from a special particle therapy to a general therapy, now. The progress in BNCT at KURRI are reported focusing on the topics for physical engineering and medical physics.

The 26th International Nuclear Physics Conference

11-16 September, 2016

Adelaide, Australia

\footnotetext{
${ }^{1}$ Speaker

(C) Copyright owned by the author(s) under the terms of the Creative Commons 


\section{Introduction}

Boron neutron capture therapy is a noninvasive therapeutic modality for treating locally invasive malignant tumors ${ }^{1}$. This therapy is known as the abbreviation, such as BNCT. It is performed according to two step procedure: (1) injection with a tumor-localizing drug containing boron. (2) irradiation with thermal or epi-thermal neutrons. In this therapy, the following nuclear reaction is used:

$$
\begin{aligned}
10 \mathrm{~B}+\mathrm{n} \rightarrow 7 \mathrm{Li}+\alpha+2.79 \mathrm{MeV} & (6.1 \%) \\
\rightarrow & 7 \mathrm{Li}^{*}+\alpha+2.31 \mathrm{MeV}(93.9 \%) \\
& \rightarrow 7 \mathrm{Li}+\gamma+0.48 \mathrm{MeV}
\end{aligned}
$$

The ranges of the $\alpha$ and the Li-7 particles generated in this reaction are approximately 8 and $5 \mu \mathrm{m}$ in tissue, respectively.

The world's first clinical irradiation using BNCT was carried out in the USA in 1951, using a neutron irradiation field installed at a research nuclear reactor ${ }^{2}$. From 1951 until 2012, BNCT has only been performed using reactor-based irradiation systems ${ }^{3-6}$. In early 2009 , the world's first accelerator-based system for BNCT clinical irradiation, Cyclotron-Based Epi-thermal Neutron Source (C-BENS) was completed at Kyoto University Research Reactor Institute (KURRI) ${ }^{7}$. The clinical trial using C-BENS was started in 2012.

At present, the development of the accelerator-based irradiation system for BNCT is energetically performed by various groups in the world ${ }^{8-11}$. It is the time when BNCT is shifting from a special particle therapy to a general therapy, now. The history and recent advances in BNCT at KURRI are reported focusing on the topics for physical engineering and medical physics.

\section{History of BNCT at KURRI}

The annual change in the accumulated number of BNCT irradiations using Heavy Water Facility installed in Kyoto University Reactor (KUR) is shown in figure 1. The first clinical study for BNCT at this facility was performed in May 1974. The BNCT clinical irradiation at this facility regularly started in February 1990. Sixty-one BNCT clinical irradiations were performed for six years until November 1995. In this period, from 1974 to 1995 , only thermal neutron irradiation could be available, so BNCT was applied to malignant melanoma and brain tumors with craniotomy.

From 1995 to 1996, Heavy Water Facility was remodeled into the Heavy Water Neutron Irradiation Facility (HWNIF) to advance $\mathrm{BNCT}^{4}$. The BNCT clinical study was restarted in November 1996 using the solo-irradiation of thermal neutrons. The mixed irradiation of thermal and epi-thermal neutrons was started in 1997. Twenty-one BNCT clinical irradiations were performed for five years until June 2001. From 1996 to 2001, BNCT was applied to malignant melanoma using thermal neutron irradiation, and brain tumors with craniotomy mainly using the mixed irradiation.

In December 2001, the first BNCT for oral cancer in the world was carried out using epithermal neutrons ${ }^{12}$. In June 2002, BNCT for brain tumors without craniotomy using epi-thermal neutrons was started ${ }^{13}$. In 2005, the wider application was promoted for the cancers of body parts such as liver, lung, and so on ${ }^{14,15}$. During five years until the KUR-operation was stopped for the 
fuel change in February 2006, one hundred and ninety-three BNCTs were carried out. During the reactor-operation stop, some minor-changes of HWNIF were carried out. In 2009, the cyclotronbased irradiation system, C-BENS was installed at KURRI ${ }^{7}$.

In May 2010, the BNCT clinical irradiation was restarted simultaneously with the restart of the reactor-operation. After the restart, two hundreds and thirty five BNCT irradiations have already been carried out as of January 2017. The clinical trial using C-BENS was also started in 2012.

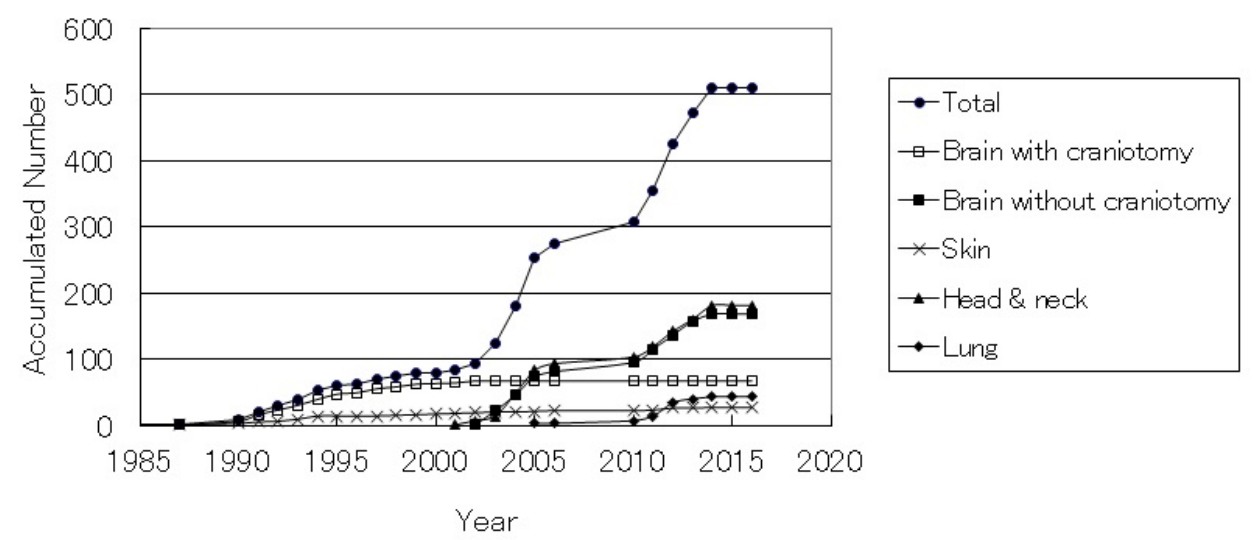

Figure 1: Annual change in the accumulated number of BNCT irradiations at KUR-HWNIF.

\section{Neutron Irradiation Systems}

Figure 2 shows the Advanced Irradiation System for BNCT, uncluding HWNIF at KUR ${ }^{4}$. Clinical irradiation utilization under the 5-MW full-power continuous KUR operation can be performed by employing both a Radiation Shielding System consisting of a shielding door and an irradiation room, and a Remote Carrying System for a patient.

The KUR-HWNIF has a heavy water tank of approximately 2-cubic-meter adjacent to the KUR core. In the heavy water tank, an aluminum-heavy water mixture, and a neutron-energy spectrum shifter of heavy water whose thickness changed from 0 to $90 \mathrm{~cm}$, are installed in order from the core side. Outside of the spectrum shifter, two thermal neutron filters of 1-mm-thick cadmium plate are installed.

The energy spectrum of the neutron beam can be controlled from almost pure thermal to epithermal within five minutes by remote control under a continuous reactor operation ${ }^{16}$. The maximum neutron flux obtained at this facility is almost $6 \times 10^{9} \mathrm{~cm}^{-1} \mathrm{~s}^{-1}$ for thermal neutrons $(<0.5$ $\mathrm{eV})$ and $9 \times 10^{8} \mathrm{~cm}^{-1} \mathrm{~s}^{-1}$ for epi-thermal neutrons $(0.5 \mathrm{eV}-10 \mathrm{keV})$.

For the irradiation system using accelerator, only the neutron source changes from reactor core to accelerator target. The basic concept is almost the same between reactor-based system and accelerator-based one. The selection for the target parts, such as nuclear reaction, kinds of accelerated particle, accelerated energy, etc. is the most important key-point. There are many ideas for the selection of neutron sources and accelerating particles and energies for $\mathrm{BNCT}^{7-11}$. Especially, the reactions of lithium or beryllium with a proton is thought to be more adequate for BCNT neutron generation because of the generated neutron energy and neutron yield. 
The nuclear reaction of ${ }^{9} \mathrm{Be}(\mathrm{p}, \mathrm{xn}){ }^{9} \mathrm{~B}$ with $30 \mathrm{-MeV}$ protons was selected for C-BENS at KURRI. Figure 3 shows an outline of the C-BENS ${ }^{7}$. The generated neutrons are moderated to thermal and epi-thermal neutrons through the moderator consisting of lead, iron, aluminum and calcium fluoride. The incident thermal neurons and gamma rays are reduced by cadmium and lead filters, respectively, and then the epi-thermal neutron beam for BNCT is obtained. In C-BENS, only epi-thermal neutron irradation is available.

In the compariaopn bewtween KUR-HWNIF and C-BENS, the beam quality of C-BENS is a little better because of the smaller contamination of fast neutrons and gamma-rays, and the epithermal neutron intensity is over two times larger than that of KUR-HWNIF. The energy spectrum of the neutron beam from C-BENS is harder than that from KUR-HWNIF. KUR-HWNIF is used for research, and C-BENS is intended for clinical use only.

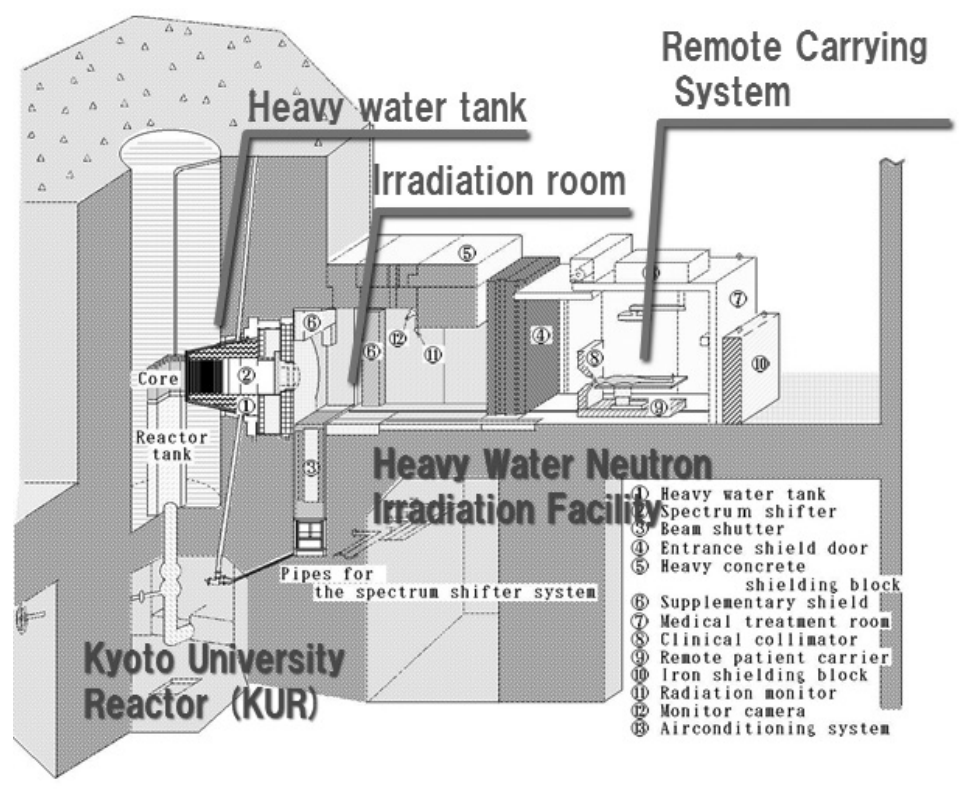

Figure 2: Outline of the Advanced Irradiation System for BNCT, installed in KUR.

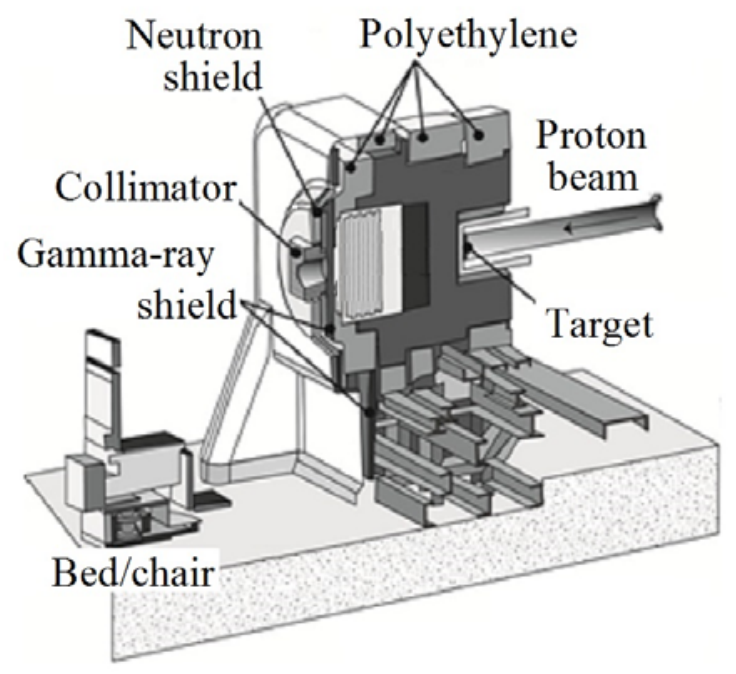

Figure 3: Outline of the Cyclotron-Based Epi-thermal Neutron Source (C-BENS). 


\section{Beam Characterization}

For the beam characterization, neutron energy spectrum is estimated by multi activation foil method, conventionally ${ }^{16}$. It is because the activation foils are small, and the irradiation field is hardly disturbed. Also, the nuclear reactions can be selected according to the neutron energy range. However, estimating the neutron energy spectrum in the $\mathrm{keV}$ range is difficult by the multi activation foil method.

In KURRI, Bonner's sphere method is applied to supplement the multi-activation foil method $^{17}$. In this method, the moderation material and wall thickness can be chosen for the neutron energy range. Activation foils (mainly for thermal neutron range) and/or He-3 counter, etc. are used as the detector. The Bonner's sphere is so large that the disturbance should be considered. The response function should be determined by simulation calculation.

Similarly to the estimation for neutron energy spectrum, the estimation at "a certain point" is necessary, in the characterization for primary gamma ray. The estimation method using small detector is better, by which the irradiation field is hardly disturbed. Thermo-luminescent dosimeter (TLD) and/or glass detector are used. Normally, TLD has sensitivity to neutrons, so the correction for the neutron response is needed. In KURRI, a specially-made TLD of $\mathrm{BeO}$ enclosed in quartz glass capsule is used ${ }^{16}$.

Ionization chambers are also used for the measurement of neutron and gamma-ray dose rates, as an online system. Four components, such as thermal neutrons $(<0.5 \mathrm{eV})$, epi-thermal neutrons $(0.5 \mathrm{eV}-10 \mathrm{keV})$, fast neutrons $(>10 \mathrm{keV})$ and gamma rays, are separately measured. Chambers are selected in considering the neutron energy spectrum and the mixing ratio of gamma ray. The wall material, wall thickness and ionization gas are changed. For example, the wall materials are $\mathrm{Si}_{3} \mathrm{~N}_{4}$, polyethylene, graphite, magnesium, etc.. As needed, a cover of Li-6-enriched LiF is prepared. The ionization gasses are $\mathrm{CO}_{2}, \mathrm{~N}_{2}$, tissue equivalent gas, $\mathrm{CH}_{4}$, Ar, etc.. When ionization chambers are used to measure the neutron dose rate, the chamber perturbation should be considered.

Recently, Multi ionization chamber system (MICS) are developed in KURRI ${ }^{18}$. Figure 4 shows an outline of MICS. The wall material, wall thickness and ionization gas are selected specially for each component, as follows: (1) a $\mathrm{Si}_{3} \mathrm{~N}_{4}$ wall and $\mathrm{N}_{2}$ gas for thermal neutrons, (2) a $\mathrm{Si}_{3} \mathrm{~N}_{4}$ wall coated with $\mathrm{LiF}$ enriched with Li-6 and $\mathrm{N}_{2}$ gas for epi-thermal neutrons, (3) a polyethylene wall and $\mathrm{CH}_{4}$ gas for fast neutrons, and (4) a graphite wall and argon gas for gammarays. Four signals from the four chambers are arithmetically operated on with Cramer's formula.

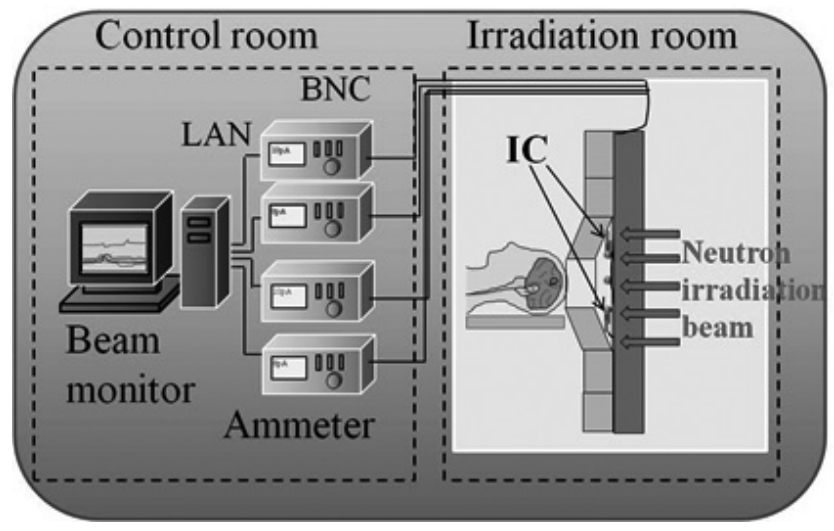

Figure 4: Outline of the multi-ionization chamber system (MICS). 
For the estimation of dose distribution in a phantom, activation foil method and TLD method are also utilized, conventionally ${ }^{19}$. Au foil with and without Cd cover, used for thermal and epithermal neutrons. Ni, In, Al, etc. are used for fast neutrons. TLD cannot be placed near the activation foils, which generates much gamma rays. Recently, some online system using ionization chambers, scintillation optical fibers (SOF), etc. are studied ${ }^{20}$.

\section{Dose Monitoring under Irradiation}

MICS can be used also as a beam monitor system. This system has almost completed, and it will be used under the BNCT irradiations at KUR-HWNIF, near future. On the patient surface, the incident epithermal neutron and the thermal neutron moderated in the patient are mixed. The separative measurement is necessary for the monitor of the incident epi-thermal neutron intensity. Activation foil method using $\mathrm{Au}$ and Mn, and TLD method are utilized, conventionally. Recently, online system using SOF detector is studied ${ }^{20}$.

The B-10 concentration is changed according to the elapsed time. The change of concentration is larger for the patients. Then, the estimation of B-10 concentration under irradiation is necessary. At present, prompt gamma-ray analysis (PGA) installed st a low-energy neutron guide tube in KUR is used ${ }^{21}$. The blood samples are obtained from the patient at some points after the injection. Then, the average B-10 concentration is estimated.

Some online estimation system for B-10 concentration has been studied. For example, prompt gamma-ray SPECT (PG-SPECT) system is studied ${ }^{22}$. In this system, the many gammaray detectors of high energy resolution, strong neutron shield, and collimator of high position resolution. The development of this system is technologically limited at present.

Gamma-ray telescope system has also been studied for the online B-10 concentration detection method. Figure 5 shows the gamma-ray telescope system installed at KUR-HWNIF ${ }^{23}$. In this telescope system, two units are installed. One is focused mainly on tumor part, and the other is only on normal part. This system is used for the liver-tumor BNCT at KURRI ${ }^{14}$.

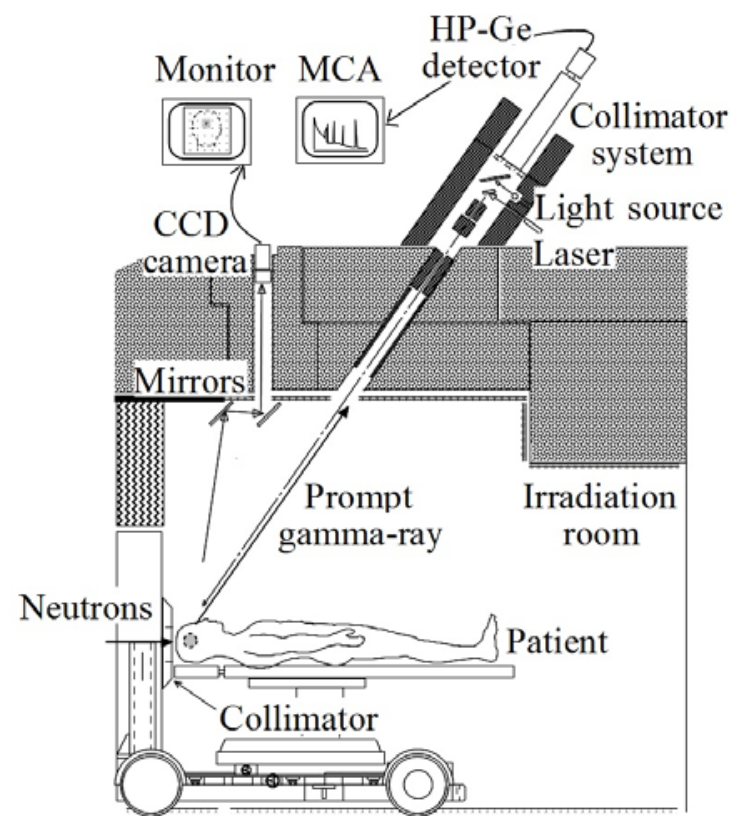

Figure 5: Outline of the gamma-ray telescope system installed at KUR-HWNIF. 


\section{Conclusion}

The recent advances in BNCT at KURRI were reported focusing on the topics for physical engineering and medical physics. Two BNCT neutron irradiation facilities in KURRI, such as KUR-HWNIF and C-BENS were introduced. Some topics for the beam characterization and online monitoring were introduced. For the more advance of BNCT, the development of the online systems are expected.

Many plans of accelerator-based BNCT systems are taking shape, but it is the situation that most of these system haven't got going yet. Recently, the shut down of the research reactor occurs successively. In the while, there is little possibility that a new research reactor is installed. At the present, BNCT is performed at a few institutes in the world, such as KURRI and Southern Tohoku General Hospital in Japan, CNEA in Argentina, NTHU in Taiwan. At the two latter institutes, only reactor-based systems are used. Shifting from reactor-based BNCT to accelerator-based BNCT, is needed to be promoted as soon as possible.

\section{Acknowledgements}

Research and clinical studies of BNCT are supported by the collaborations of several universities and institutes such as the Osaka Medical College, Kawasaki Medical School, Osaka University, University of Tsukuba, and Southern Tohoku General Hospital. The authors thank all of these collaborators.

\section{References}

[1] G. L. Locher, Am. J. Roentgenol. 36, 1 (1936)

[2] L. E. Farr, et al., Am. J. Roentgenol. 71, 279 (1954)

[3] O. K. Harling, et al., Nucl. Sci. Eng. 110, 330 (1992)

[4] Y. Sakurai and T. Kobayashi, Nucl. Instr. Meth. A 453, 569 (2000)

[5] S. J. Gonzalez, et al., Appl. Radiat. Isot. 61, 1101 (2004)

[6] L. W. Wang, et al., Appl. Radiat. Isot. 69, 1803 (2011)

[7] H. Tanaka, et al., Nucl. Instr. Meth. B 267, 1970 (2009)

[8] C. N. Culbertson, et al., Appl. Radiat. Isot. 61, 733 (2004)

[9] C. Ceballos, et al., Appl. Radiat. Isot. 69, 1660 (2011)

[10] A. J. Kreiner, et al., Appl. Radiat. Isot. 69, 1672 (2011)

[11] H. Kumada, et al., Appl. Radiat. Isot. 88, 211 (2014)

[12] I. Kato, et al., Appl. Radiat. Isot. 67, S37 (2009)

[13] S. Kawabata, et al., Appl. Radiat. Isot. 67, S15 (2009)

[14] M. Suzuki, et al., Jpn. J. Clin. Oncol. 37, 376 (2007)

[15] M. Suzuki, et al., Radiother. Oncol. 88, 192 (2008)

[16] Y. Sakurai and T. Kobayashi, Nucl. Instr. Meth. A 531, 585 (2004) 
[17] H. Ueda, et al., Appl. Radiat. Isot. 69, 1657 (2011)

[18] T. Fujii, et al., Appl. Radiat. Isot. 69, 1862 (2011)

[19] Y. Sakurai and T. Kobayashi, Med. Phys. 29, 2328 (2002)

[20] M. Ishikawa, et al., Appl. Radiat. Isot. 61, 775 (2004)

[21] T. Kobayashi and K. Kanda, Nucl. Instr. Meth. 204, 525 (1983)

[22] T. Kobayashi, et al., Med. Phys. 27, 2124 (2000)

[23] Y. Sakurai, et al., Appl. Radiat. Isot. 61, 829 (2004) 